\title{
The Exploration of the Outer Planets
}

THE discovery by Kemp and his colleagues (see page 169) of circular polarization on Jupiter is a reminder not merely of the problems of the outer planets which have been crying out for a solution for several years but also that much will be done in the years ahead to define problems as yet unrecognized. Classical methods of observation will no doubt contribute in important ways, but it is not too soon to look forward to the spate of information that will presumably be produced by the Grand Tour of the outer planets, a reconnaissance mission which is currently being planned by NASA. The gravity field of Jupiter would be used to deflect each spacecraft toward two more outer planets. Spacecraft launched in 1976 and 1977 would go past Jupiter to Saturn and Pluto. Spacecraft launched in 1979 would encounter Uranus and Neptune, after leaving Jupiter. Because the last encounter would take place at extremely great distances (30 AU), the typical mission would last about ten years.

The mission will investigate fundamental questions regarcing the origin and evolution of the solar system. The little that is known about the outer planets shows them to be very unlike the Earth or its nearest companions, Venus and Mars. Although very large, the outer planets are less dense than our own Moon, and it thus seems likely that they are composed principally of hydrogen, perhaps in a fluid or even a solid state. Their composition is believed to approximate closely that of the primordial nebulae from which the Sun and planets presumably accumulated. Information regarding their composition could thus throw some light on the abundance of the elements in the original solar nebula.

The large size and mass of the outer planets have retarded the loss of their atmospheres, unlike the inner planets the atmospheres of which were too hot and gravity fields too weak to retain their primordial atmospheres. Furthermore, the Earth's atmosphere has been drastically modified by life processes. Thus, the atmospheres of the terrestrial planets are almost devoid of hydrogen and helium, which are expected to be abundant in the atmospheres of the outer planets. One goal of the mission would undoubtedly be to measure the ratio of hydrogen and helium at all the giant planets. This could be accomplished by measurements made in diverse parts of the electromagnetic spectrum such as the amount of solar ultraviolet scattered by the upper atmosphere, the infrared radiance from the lower atmosphere, and the dispersion of the radio waves using the radio signals of the spacecraft.

The outer planets may retain other traces of the early history of the solar system. The Earth's atmosphere must originally have been similar in composition to the present atmospheres of Jupiter and Saturn. Complex organic molecules may be formed in such atmospheres and on Earth may have been precursors to life. Such molecules have been invoked to explain the unusual colouring of the clouds that cover Jupiter and Saturn. Organic molecules may be created in hydrogen-rich atmospheres, perhaps by the passage of a lightning discharge. Simple detectors on spacecraft passing near Jupiter and Saturn could investigate the possible occurrence of lightning, and more complex infrared instruments could investigate the origin of the colours often seen in the clouds.

The measurement of infrared emissions from the atmosphere has other important implications for outer planet research. Earth-based measurements indicate that both Jupiter and Saturn have an internal source of energy and they radiate significantly more energy than they absorb from the Sun. It is possible that both planets are contracting slowly and gravitational energy is being converted into heat, but this question cannot be answered from Earth because of the inability of an Earth-based observer to view the dark side of the outer planets. Careful measurements by spacecraft of the relative amounts of energy reflected from the planets in the visible and radiated into the infrared may settle this question.

The outer planets apparently differ from the terrestrial planets other than Earth in yet another way. Earth is the only inner planet having a significant magnetic field and trapped radiation. Radio astronomical observations at long wavelengths indicate that Jupiter is one of the brightest radio sources in the sky. Studies of these intense planetary emissions, and those at shorter decimetre wavelengths, imply that Jupiter has a planetary magnetic field at least ten times stronger at its surface than the Earth's field. The field is so intense and the planet is so large that the magnetic field of Jupiter occupies an enormous volume relative to that in which the Earth's radiation belts are confined. The Jovian radio noise is undoubtedly radiated by energetic electrons trapped in this voluminous magnetic sphere of influence.

Direct measurements of trapped radiation near other planets are probably required in order to understand fully the Earth's radiation belts. The origin of the particles and their subsequent acceleration to high energies is still a matter of keen scientific interest. The trapped energetic particles seem to be extracted by some mechanism from the solar wind and the ionized gas escapes continuously from the Sun's corona, which fills the inner solar system. One of the scientific studies carried out at the outer planets would undoubtedly be the nature of their interaction with the solar wind. Furthermore, the properties of the Jovian trapped particles could be measured, and trapped radiation at the other giant planets could be sought by particle detectors. Particle trapping in large scale magnetic fields seems to be commonplace in the universe, as witness their role in recent attempts to explain pulsars.

A favourable conjunction of the outer planets making multiplanet missions of this kind possible occurs roughly every 175 years. Will the United States celebrate its 200th anniversary in 1976 by embarking on this ambitious undertaking? There are obviously many scientists in Britain and Europe who are willing to join the celebration by becoming involved in the mission. Although the interval between the first and last encounter would be eleven years, the set of four launches presently being studied would imply a rendezvous with at least one of the outer planets in seven of those years. Such a prospect would maintain a high level of interest in outer planet research throughout the world. 